\title{
High expression of PIWIL2 promotes tumor cell proliferation, migration and predicts a poor prognosis in glioma
}

\author{
JINQUAN LI ${ }^{*}$, LI XU ${ }^{1 *}$, ZHEN BAO $^{1}$, PENG XU $^{2}$, HAO CHANG $^{3}$, JINGJING WU $^{4}$, \\ YUANQI BEI $^{2}$, LIUWAN XIA ${ }^{2}$, PEIZHANG WU ${ }^{2}$ and GANG CUI ${ }^{1}$ \\ ${ }^{1}$ Department of Neurosurgery, The First Affiliated Hospital of Soochow University, Suzhou, Jiangsu 215006; \\ 2 Jiangsu Province Key Laboratory for Inflammation and Molecular Drug Targets, Nantong University, Nantong, \\ Jiangsu 226001; ${ }^{3}$ Department of Neurosurgery, Affiliated Wuxi Second Hospital of Nanjing Medical University, \\ Wuxi, Jiangsu 214002; ${ }^{4}$ Department of Oncology, Nantong Rich Hospital, Nantong, Jiangsu 226001, P.R. China
}

Received October 19, 2016; Accepted December 5, 2016

DOI: $10.3892 /$ or.2017.5647

\begin{abstract}
Piwi-like RNA-mediated gene silencing 2 (PIWIL2), has been reported as an oncogene tightly associated with the genesis and progression of various malignancies. Nevertheless, the function of the PIWIL2 protein in human gliomas has not yet been clarified. In this study, we sought to investigate the clinical significance of PIWIL2 expression and reveal its function in the pathological process of gliomas. Through western blot and immunohistochemical analyses we found that PIWIL2 was overexpressed in glioma tissues. Moreover, the expression level of PIWIL2 was also significantly correlated with the WHO grades of human gliomas and Ki-67 expression. Kaplan-Meier curves indicated that PIWIL2 was a prognostic factor for the survival of glioma patients and a high expression of PIWIL2 was correlated with a poor prognosis. In vitro, knockdown of PIWIL2 in glioma cells was shown to induce cell cycle arrest and increase apoptosis. Furthermore, silencing of PIWIL2 expression also obviously suppressed the migration of glioma cells. All the results demonstrated that PIWIL2 plays a significant role in the pathogenesis of human gliomas and may be used as a potential diagnostic marker and a therapeutic target of glioma in the future.
\end{abstract}

\section{Introduction}

Human malignant glioma, as the most common primary central nervous system tumor, has an adverse prognosis for patients $(1,2)$. Even with a variety of treatments, patients affected by glioblastoma multiforme (GBM), which is

Correspondence to: Professor Gang Cui, Department of Neurosurgery, The First Affiliated Hospital of Soochow University, Suzhou, Jiangsu 215006, P.R. China

E-mail: sdfyycg@163.com

${ }^{*}$ Contributed equally

Key words: glioma, PIWIL2, proliferation, migration, prognosis regarded as the highest malignant degree of astrocytic tumors (WHO grade IV), survive only 12 months on average after diagnosis (3). Unfortunately, despite enormous advances in neurosurgery and chemoradiotherapy in recent years, the prognosis of malignant gliomas is still poor. The rapid proliferation of glioma cells and their strong invasive ability are the critical reasons for the resistance of conventional treatments $(4,5)$. Therefore, it is urgent to explore the molecular pathological mechanism concerning the occurrence and development of gliomas, and to apply specific molecules that may be invoked as new diagnostic markers and therapeutic targets.

Piwi-like RNA-mediated gene silencing 2 (PIWIL2) protein is a member of the Argonaute family (6). Argonaute proteins, as a highly conserved protein family, are subdivided into two subfamilies, Piwi and Ago (7). The Piwi subfamilies characterized by highly conserved Piwi/Argonaute/Zwille (PAZ) and P-element induced wimpytestis (PIWI) domains, were found to play critical roles in stem cell self-renewal, RNA interference, spermatogenesis, chromatin remodeling and translational regulation $(6,8,9)$. In humans, the Ago/Piwi family is comprised of PIWIL1 (HIWI), PIWIL2 (HILI, CT80, Miwi like), PIWIL3 (HIWI3), and PIWIL4 (HIWI2) (10). Among the PIWI subfamily members, PIWIL2 has been demonstrated to play an important role in the pathological process of various malignant tumors (11). It has been reported that PIWIL2 is stably expressed in precancerous stem cells (pCSCs) in breast cancer, and may participate in the regulation of pCSC development and differentiation (12). Moreover, PIWIL2 was found to be mainly expressed in breast cancer stem cells, and inhibited their apoptosis through the Stat3/Bcl-XL pathway, and thus could be used as a biomarker for breast cancer $(13,14)$. In addition, recent research also indicated that PIWIL2 is highly expressed in lung, gastric, bladder, prostate, colorectal and papillary thyroid cancer, and is also closely associated with the initiation, development and metastasis of tumors (15-18). Notwithstanding the research of PIWIL2 in diverse human cancers which has yielded fruitful results, we also detected the expression of PIWIL2 in glioma tissues and cells. Yet, its function and mechanism in the occurrence and development of glioma still remain to be clarified by further research. 
In the present study, we ascertained the high expression of PIWIL2 in human glioma tissues and cell lines, and indicated that overexpression of PIWIL2 predicts a poor prognosis in patients with glioma. Furthermore, we explored the possible impact of PIWIL2 on cellular proliferation and migration in U87 glioma cells. Our research demonstrated that PIWIL2 plays an important role in the pathological process of human gliomas, and we expect to provide a novel molecular target for glioma diagnosis and therapies in the future.

\section{Materials and methods}

Patients and tissue specimens. A total of 97 glioma specimens (including WHO grade II-IV) was collected from patients diagnosed with glioma for the first time and who had received no prior therapy. All the tissues were obtained from the Affiliated Hospital of Nantong University between 2011-2015 and their clinicopathological data were provided by the Department of Pathology. Normal brain tissue was donated by patients who had succumbed to traffic accidents and were ascertained to be without any pathological changes. All the tissues were collected and applied in accordance with the ethical standards of the World Medical Association and all of the studies were approved by the Ethics Committee of the Affiliated Hospital of Nantong University. Specimens used for immunoblot analysis were frozen in liquid nitrogen immediately after surgical removal, and then stored at $-80^{\circ} \mathrm{C}$. Some specimens were fixed in $10 \%$ formalin before they were sliced into paraffin sections for immunohistochemical analysis.

Cell culture. The human glioma cell lines H4, A172, U251, U87 and U118 were purchased from the Cell Library of the Chinese Academy of Sciences. All the cells were cultured in Dulbecco's modified Eagle's medium (DMEM; Gibco-BRL, Grand Island, NY, USA) with $10 \%$ fetal bovine serum (FBS), $2 \mathrm{mM}$ L-glutamine and $100 \mathrm{U} / \mathrm{ml}$ of a penicillin/streptomycin mixture (Gibco-BRL) at $37^{\circ} \mathrm{C}$, and $5 \% \mathrm{CO}_{2}$.

Antibodies. The antibodies used for the western blot analysis were as follows: anti-PIWIL2 (diluted 1:500), anti-proliferating cell nuclear antigen (PCNA, diluted 1:500), anti-p53 (diluted 1:500), anti-p21 (diluted 1:500), anti-cyclin-dependent kinase 2 (CDK2, diluted 1:500), anti-cyclin D1 (diluted 1:1,000), and anti-cyclin E polyclonal antibody (diluted 1:500) (all from Santa Cruz Biotechnology, CA, USA), anti-cleaved caspase 3 (diluted 1:200; Cell Signaling Technology, Danvers, MA, USA), anti-B-cell CLL/lymphoma 2 (Bcl-2, diluted 1:1,000), anti-glyceraldehyde-3-phosphate dehydrogenase (GAPDH, diluted 1:1,000), anti-N-cadherin (diluted 1:1,000), anti-Ecadherin (diluted 1:1,000), anti-vimentin (diluted 1:1,000), and anti-matrix metallopeptidase 9 (MMP9 diluted 1:1,000) (all from Santa Cruz Biotechnology). The antibodies used for immunohistochemistry were: anti-PIWIL2 (diluted 1:200), and anti-Ki-67 (1:300) (both from Santa Cruz Biotechnology).

Immunohistochemical staining. Glioma tissues were fixed in $10 \%$ formalin, and then embedded in paraffin to produce paraffin sections. The glioma paraffin sections with xylene dewaxing were rehydrated in graded ethanol. Then, we used $0.3 \%$ hydrogen peroxide to block endogenous peroxidase activity. The sections were then processed in $0.1 \mathrm{M}$ citrate buffer $(\mathrm{pH} 6.0)$ and heated to $120^{\circ} \mathrm{C}$ in an autoclave for $5 \mathrm{~min}$ for antigen retrieval. As the sections cooled, they were rinsed in the phosphate-buffered saline (PBS) $(\mathrm{pH} 7.2)$, then incubated with $5 \%$ bovine serum $1.5 \mathrm{~h}$ at room temperature to block nonspecific reactions. The tissue sections were incubated with anti-PIWIL2 antibody and anti-Ki-67 antibody overnight at $4^{\circ} \mathrm{C}$. Meanwhile, the same concentration of non-specific immunoglobulin IgG (Sigma Chemical Co., St. Louis, MO, USA) was used as the first antibody for the negative control group. According to the manufacturer's instructions, the horseradish peroxidase-conjugated anti-rabbit or anti-mouse Ig polymer was used as a second antibody (EnVision kit; Dako) to incubate the tissue sections for $30 \mathrm{~min}$ at room temperature. After being rinsed in PBS, the slides were counterstained with hematoxylin, dehydrated, and mounted with resin.

Immunohistochemical analysis. Without any knowledge of the clinicopathological characteristics of the patients, all of the immunohistochemical staining sections were evaluated by three pathologists in a blinded manner, and discrepancies were resolved by consensus. At least five high-power fields (Leica microscope; Leica, Wetzlar, Hessen, Germany) were chosen randomly in each specimen, and more than 300 cells in each field were counted to determine the mean percentage of signal-positive cells. In order to evaluated the expression of PIWIL2 and Ki-67 in glioma, the staining intensity score and the proportion of positive tumor cells were both graded with different scores, and the product of the two scores was used to evaluate the staining results. An intensity score representing the average intensity of positive cells: 0 (negative staining); 1 (weak staining: light yellow); 2 (moderate staining: yellow brown) and 3 (strong staining: brown), and the percentage of tumor cells stained positive were scored as follows: 0 (0-5\% tumor cells stained), 1 (6-25\% tumor cells stained), 2 (26-50\% tumor cells stained), 3 (51-75\% tumor cells stained) and 4 (76-100\% tumor cells stained). Then intensity and staining scores were multiplied to create an immunoreactivity score (IS), and the IS was further divided as follows: 0-1 (-); 2-4 (+); 5-7 (++); >8 (+++). For statistical analysis, '-' and ' + ' were considered low levels of immunoreactivity, while ' ++ ' and '+++' were considered high levels of immunoreactivity (19).

Western blot analysis. Glioma tissue samples and cell protein were homogenized in a homogenization buffer $(1 \mathrm{M}$ TrisHCl $\mathrm{pH} 7.5,1 \%$ Triton $\mathrm{X}-100,1 \%$ Nonidet $\mathrm{p}-40,10 \%$ sodium dodecyl sulfate, $0.5 \%$ sodium deoxycholate, $0.5 \mathrm{M}$ EDTA, $10 \mu \mathrm{g} / \mathrm{ml}$ leupeptin, $10 \mu \mathrm{g} / \mathrm{ml}$ aprotinin, and $1 \mathrm{mM}$ PMSF) on the ice, and centrifuged at $13,000 \mathrm{x}$ g for $10 \mathrm{~min}$ at $4^{\circ} \mathrm{C}$ to collect the supernatant liquid. Then the samples were denatured at $100^{\circ} \mathrm{C}$ for $15 \mathrm{~min}$ and used immediately or stored at $-20^{\circ} \mathrm{C}$. Protein concentrations were assessed with a Bio-Rad protein assay (Bio-Rad, Hercules, CA, USA). The protein extracts were separated by sodium dodecyl sulfate-polyacrylamide gel electrophoresis (SDS-PAGE) and transferred to polyvinylidene difluoride filter (PVDF) membranes (Millipore, Bedford, MA, USA). Then the membranes were blocked in 5\% fat-free milk with TBST (150 mM NaCl, $20 \mathrm{mM}$ Tris, $0.05 \%$ Tween-20) for $2 \mathrm{~h}$ at room temperature, and incubated with the corresponding primary antibodies for $6-8 \mathrm{~h}$ at $4^{\circ} \mathrm{C}$. Subsequently, 
the membranes were rinsed with TBST three times, 5 min each time, and then incubated with horseradish peroxidase-linked IgG as the secondary antibody for $2 \mathrm{~h}$ at room temperature. Finally, the membranes were detected by a computer imaging system (Imaging Technology, Ontario, Canada). There were at least three independent experiments performed to ensure the rigorousness of the results.

Transient transfection. The PIWIL2-shRNAs were designed and synthesized by GenechemCo. Ltd. (Shanghai, China). Full-length PIWIL2 was amplified using PCR and subcloned into pcDNA3.1 construct (Invitrogen, Carlsbad, CA, USA). PIWIL2-shRNA\#1 target sequence was: 5'-ACCGGC UGGGUUGAACUAAA-3', PIWIL2-shRNA\#2 target sequence was: 5'-ACAGCCUCAAACACUGUGCAC-3', and control-shRNA target sequence was: 5'-GUACCGCAC GUCAUUCGUAUC-3'. The glioma cells were transfected with PIWIL2-shRNAs or negative-control shRNA using Lipofectamine 2000 (Invitrogen) according to the manufacturer's instructions, and the corresponding glioma cells without any treatment were used as mock groups.

Flow cytometric analysis of cell apoptosis and the cell cycle. For the cell apoptosis analysis, U87 cells transfected with PIWIL2-shRNAs and negative control-shRNA were cultured for $48 \mathrm{~h}$. PE Annexin V apoptosis detection kit I (BD Pharmingen, San Jose, CA, USA) and flow cytometer BD FACScan (Becton Dickinson, San Jose, CA, USA) were used in conjunction according to the manufacturer's instructions to detect apoptosis. For the cell cycle analysis, after transfection for $48 \mathrm{~h}$, U87 cells were collected and fixed with $70 \%$ ethanol for $24 \mathrm{~h}$ at $-4^{\circ} \mathrm{C}$. Then the cells were rinsed with cold PBS and incubated with $10 \mathrm{mg} / \mathrm{ml}$ PI and $0.5 \mathrm{mg} / \mathrm{ml} \mathrm{RNase} \mathrm{A} \mathrm{for}$ $20 \mathrm{~min}$ at $37^{\circ} \mathrm{C}$. The DNA contents of the labeled cells were analyzed by a Becton Dickinson flow cytometer BD FACScan (Becton Dickinson).

Colony formation assay. U87 cells transfected with PIWIL2-shRNA and negative-control shRNA were seeded in 6 -cm culture plates $(2,000$ cells/plate). After being cultured for 14 days at $37^{\circ} \mathrm{C}$, the colonies were washed with PBS, fixed with methanol for $30 \mathrm{~min}$, and stained with $0.5 \%$ crystal violet (Solarbio, Beijing, China) for $30 \mathrm{~min}$. Then, the number of colonies was counted under a microscope, and colonies containing $>50$ cells were considered as positive for growth.

Wound healing assay. U87 cells transfected with PIWIL2-shRNA\#1 or control-shRNA, respectively, were seeded in 6-well plates and grown to confluence. Subsequently, the cells were serum starved for $12 \mathrm{~h}$, and scratched on the monolayer by using a sterile $10-\mu 1$ micropipette tip. Then the U87 cells were washed twice with PBS, and cultured in serum-free DMEM medium at $37^{\circ} \mathrm{C}$ and $5 \% \mathrm{CO}_{2}$, for an additional $24 \mathrm{~h}$. Images were captured using an inverted Leica phase contrast microscope (Leica DFC 300 FX) at 0- and 24-h time-points.

Transwell migration assay. U87 cells transfected with PIWIL2-shRNA\#1 and negative-control shRNA were suspended in DMEM containing $0.1 \%$ bovine serum albumin, respectively. Cells $\left(1 \times 10^{5}\right)$ were added to the top chambers of 24-well Transwell plates (8- $\mu \mathrm{m}$ pore size; Corning), and DMEM containing $10 \%$ FBS was added to the bottom chambers. Following incubation at $37^{\circ} \mathrm{C}$ for $24 \mathrm{~h}$, the cells which migrated to the bottom chambers were fixed with paraformaldehyde and stained with crystal violet. The number of migrating cells in 5 fields were counted under an x 200 magnification for all the chambers. Experimental data were obtained from triplicate chambers and the experiments were repeated three times.

Statistical analysis. The statistical correlations between PIWIL2 and Ki-67 expression and the clinicopathological features were analyzed by the Chi-square $\left(\chi^{2}\right)$ test or Fisher's exact test. Kaplan-Meier survival plots were constructed to analyze the survival rate of patients with glioma, and the log-rank test was performed to compare the curves. Statistical analysis was performed using SPSS 22.0 statistical software (SPSS, Inc., Chicago, IL, USA). All values are expressed as the mean \pm SEM, and $\mathrm{p}<0.05$ was considered to be statistically significant.

\section{Results}

PIWIL2 overexpression in glioma tissues. In order to explore the possible role of PIWIL2 in the progression of glioma, we first evaluated the expression of PIWIL2 in two normal brain specimens and three pairs of glioma specimens from different pathological grades (WHO grade II-IV) by western blot analysis. It was found that PIWIL2 was overexpressed in the glioma tissues, and its expression level exhibited an increasing trend from low-grade glioma tissues to high-grade glioma tissues (Fig. 1A and B). Furthermore, the expression of PIWIL2 and proliferation marker Ki-67 (20) were examined in 97 glioma tissue samples by immunohistochemistry. Similar to the expression of Ki-67, PIWIL2 was highly expressed in the glioma samples with a high degree of malignancy and its expression was significantly higher than that in the low-grade malignancy samples (Fig. 1C).

Correlation between the expression of PIWIL2 and the survival of glioma patients. According to the results obtained with immunohistochemical analysis, the data revealed that PIWIL2 and Ki-67 were expressed with a positive correlation in glioma patients (Fig. 1D). Subsequently, we further analyzed the immunohistochemical results and the clinicopathological data of 97 patients with glioma to explore the clinical significance of PIWIL2 expression (Table I). We observed that the expression of PIWIL2 was clearly associated with the clinicpathological grade of the glioma patients $(\mathrm{P}=0.001)$ and Ki-67 expression $(\mathrm{P}=0.001)$, but there was no obvious correlation between the expression of PIWIL2 and other clinicopathological patient factors such as age $(\mathrm{P}=0.577)$, sex $(\mathrm{P}=0.443)$, tumor location $(\mathrm{P}=0.848)$, tumor size $(\mathrm{P}=0.800)$ and extent of resection $(\mathrm{P}=0.626)$. Subsequently, Cox's proportional hazards model was constructed and it revealed that a high expression level of PIWIL2 and WHO grade were prognostic indicators of the survival time of the glioma patients $(\mathrm{P}<0.05$, Table II). By using Kaplan-Meier survival curves, we observed that the expression of PIWIL2 was negatively correlated with the overall survival of the glioma patients (Fig. 1E and F). In 

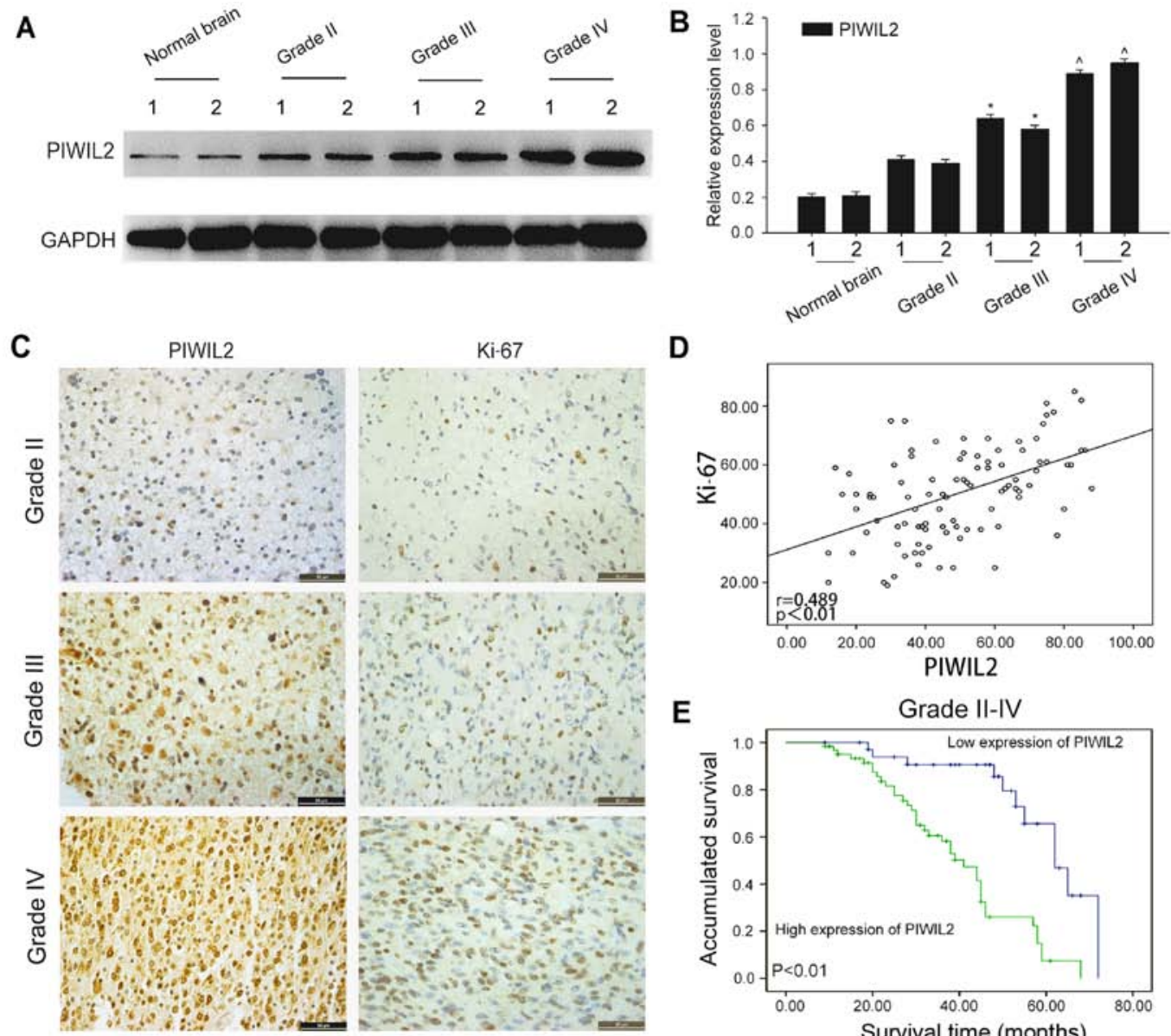

D

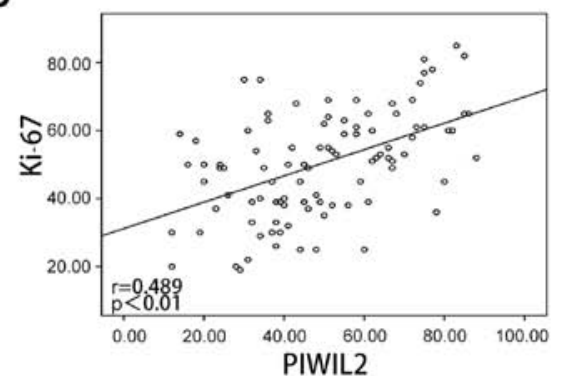

E

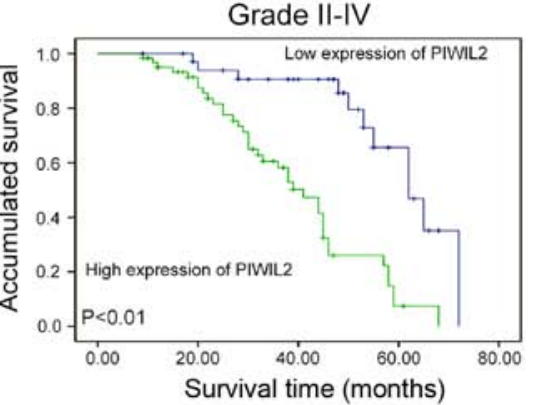

$\mathbf{F}$

Grade II
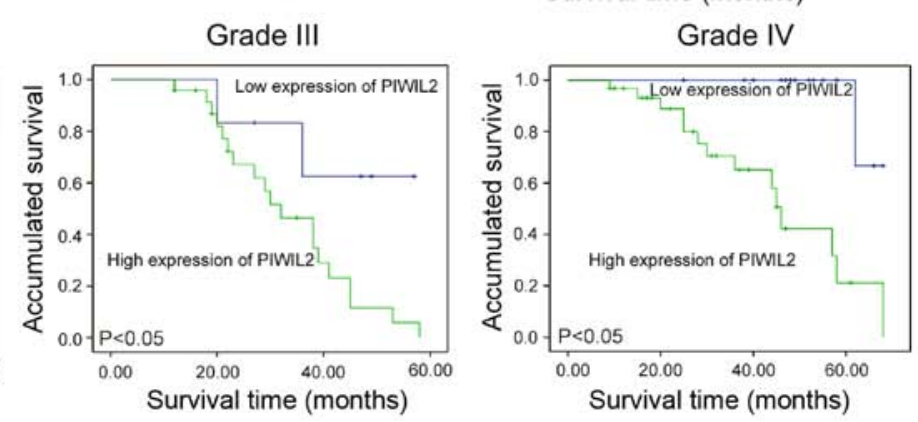

Figure 1. Relationship between Piwi-like RNA-mediated gene silencing 2 (PIWIL2) and glioma pathological grade, Ki-67 and glioma prognosis. (A) The protein expression levels of PIWIL2 were detected in glioma tissue of different grades (grades II-IV) and normal brain tissues by western blot analysis Glyceraldehyde-3-phosphate dehydrogenase (GAPDH) was used as a cross-reference. (B) The bar graph displays the ratio of PIWIL2 protein to GADPH for the aforementioned tissues by densitometry. The data are expressed as the mean \pm SEM of three independent experiments $\left(n=3,{ }^{*}, p<0.01\right)$. (C) Immunohistochemical staining. Paraffin-embedded glioma tissue sections (grades II, III, and IV) were stained with anti-PIWIL2 antibodies and anti-Ki-67 antibodies, and then counterstained with hematoxylin (magnification, x400; scale bar, $50 \mu \mathrm{m}$ ). (D) The correlation between PIWIL2 and Ki-67. (E and F) Kaplan-Meier survival curves of 97 glioma patients with high PIWIL2 expression vs. low PIWIL2 expression. Based on the mean PIWIL2 percentages, patients were divided into a high PIWIL2 expression group and a low PIWIL2 expression group. According to the WHO grades, the patients were divided into three groups (WHO grade II, III and IV). Patients in the high-expression PIWIL2 group had a significantly shorter overall survival $(\mathrm{P}<0.05)$.

summary, we conclude that a high expression of PIWIL2 was an important determinant of poor prognosis in patients with glioma.

Expression of PIWIL2 in human glioma cell lines and the promotion of $G 1 / S$ phase transition. In our preliminary investigation, we determined that the expression of PIWIL2 was positively correlated with the expression of Ki-67 in glioma specimens. Thus, we hypothesized that PIWIL2 may be involved in the cell cycle progression of glioma cells. Due to the fact that PIWIL2 is expressed at the highest level in
U87 cells, we selected it as our main experimental cell line in the following experiments (Fig. 2A and B). In order to ascertain our conjecture, we built a serum starvation and re-feeding model. U87 cells were controlled in the serum-free environment for $72 \mathrm{~h}$, and the results of the flow cytometric analysis demonstrated that the highest proportion of U87 cells was arrested in the G0/G1 phase. Subsequently, we added $10 \%$ FBS and harvested the cells at 0, 4, 8, 12 and $24 \mathrm{~h}$. Flow cytometric analysis revealed that most U87 cells shifted from the G0/G1 phase to the $S$ phase gradually with the readdition of serum (Fig. 2C). Meanwhile, we detected changes in the 
Table I. PIWIL2 expression and clinicopathological variables in 97 glioma specimens.

\begin{tabular}{|c|c|c|c|c|c|}
\hline \multirow[b]{2}{*}{ Variables } & \multirow[b]{2}{*}{ Total } & \multicolumn{2}{|c|}{$\begin{array}{l}\text { PIWIL2 } \\
\text { expression }\end{array}$} & \multirow[b]{2}{*}{$\chi^{2}$ value } & \multirow[b]{2}{*}{ P-value } \\
\hline & & Low & High & & \\
\hline \multicolumn{6}{|l|}{ Age (years) } \\
\hline$<50$ & 59 & 20 & 39 & 0.312 & 0.577 \\
\hline$\geq 50$ & 38 & 15 & 23 & & \\
\hline \multicolumn{6}{|l|}{ Sex } \\
\hline Male & 56 & 22 & 34 & 0.589 & 0.443 \\
\hline Female & 41 & 13 & 28 & & \\
\hline \multicolumn{6}{|l|}{ Tumor location } \\
\hline Frontal & 23 & 10 & 13 & 1.379 & 0.848 \\
\hline Parietal & 9 & 2 & 7 & & \\
\hline Occipital & 21 & 7 & 14 & & \\
\hline Temporal & 17 & 6 & 11 & & \\
\hline Unknown & 27 & 10 & 17 & & \\
\hline \multicolumn{6}{|l|}{ Tumor size $(\mathrm{cm})$} \\
\hline$<4$ & 51 & 19 & 32 & 0.064 & 0.800 \\
\hline$\geq 4$ & 46 & 16 & 30 & & \\
\hline \multicolumn{6}{|l|}{ Extent of resection } \\
\hline Total resection & 33 & 13 & 20 & 0.238 & 0.626 \\
\hline Subtotal resection & 64 & 22 & 42 & & \\
\hline \multicolumn{6}{|l|}{ WHO grade } \\
\hline II & 22 & 15 & 7 & 13.675 & $0.001^{\mathrm{a}}$ \\
\hline III & 30 & 6 & 24 & & \\
\hline IV & 45 & 14 & 31 & & \\
\hline \multicolumn{6}{|l|}{$\mathrm{Ki}-67$} \\
\hline Low expression & 28 & 17 & 11 & 10.355 & $0.001^{\mathrm{a}}$ \\
\hline High expression & 69 & 18 & 51 & & \\
\hline
\end{tabular}

Statistical analyses were performed using the Pearson $\chi^{2}$ test. ${ }^{a} \mathrm{P}<0.05$ was considered significant. PIWIL2, Piwi-like RNA-mediated gene silencing 2.

expression levels of PIWIL2 at different time-points during cell progression. Furthermore, the expression of two cell cycle-related proliferation markers, PCNA and cyclin D1 (21), were assessed by western blot analysis at the corresponding time-points. As expected, we found that the expression of PIWIL2 increased gradually after serum readdition, and a similar trend was obtained with these cell cycle-related proliferation markers (Fig. 2D and E). Taking into consideration the aforementioned experimental results, we put forward a preliminary conclusion that PIWIL2 may be related to the proliferation of glioma in a cell cycle-dependent pathway.

Downregulation of PIWIL2 inhibits cell growth and induces the delay of $G 1 / S$ transition. To further clarify the function of PIWIL2, we investigated the effect of the interference of PIWIL2 expression on the proliferation of glioma cells. We transfected U87 cells with PIWIL2-shRNAs or control-shRNA, respectively, and then detected the expression
Table II. Contribution of various potential prognostic factors to survival by Cox regression analysis in 97 glioma specimens.

\begin{tabular}{lccr}
\hline Characteristic & Hazard ratio & $95 \%$ CI & P-value \\
\hline Age & 1.157 & $0.643-2.081$ & 0.627 \\
Sex & 0.765 & $0.429-1.366$ & 0.365 \\
Tumor location & 1.018 & $0.844-1.226$ & 0.855 \\
Tumor size & 0.902 & $0.505-1.611$ & 0.727 \\
WHO grade & 2.792 & $1.708-4.562$ & $<0.001^{\mathrm{a}}$ \\
PIWIL2 expression & 4.780 & $2.320-9.852$ & $<0.001^{\mathrm{a}}$ \\
Ki-67 expression & 2.865 & $1.450-5.662$ & $0.002^{\mathrm{a}}$ \\
\hline
\end{tabular}

Statistical analyses were performed using Cox regression analysis. CI, confidence interval; PIWIL2, Piwi-like RNA-mediated gene silencing 2 . ${ }^{\mathrm{a}}<0.05$ was considered significant.

of PIWIL2 protein by western blot analysis to determine the effectiveness of all the PIWIL2-shRNAs (Fig. 3A and B). The results revealed that PIWIL2-shRNA\#1 exhibited the most effective decrease, thus we selected it as our main shRNA in the following experiments. In comparison to the control group, flow cytometry indicated that a larger proportion of U87 cells transfected with PIWIL2-shRNA\#1 were arrested in the G0/G1 phase, which meant that PIWIL2 may contribute to the G1/S phase transition of U87 cells (Fig. 3C and D). Previous studies demonstrated that PIWIL2 could facilitate tumor cell (HeLa and HepG2 cells) transformation from the G0/G1 phase to the $\mathrm{S}$ phase (22). Moreover, it could also inhibit the expression of p53, which is considered to be an important regulator of the cell cycle (11). In our study, western blot analysis indicated that silencing of PIWIL2 promoted the expression of p53 and p21, and inhibited the expression of cyclin D1 and CDK2 concomitantly (Fig. 3E and F). Furthermore, colony formation assay revealed that the proliferation of U87 glioma cells was significantly suppressed after PIWIL2 knockdown (Fig. 3G). These results demonstrated that PIWIL2 may regulate the cell cycle via dysregulation of the p53-p21-cyclin E/Cdk2 pathway, and interfering with its expression could block glioma cell cycle progression, and then inhibit its proliferation.

Knockdown of PIWIL2 expression induces apoptosis in glioma cells. A decrease in of apoptosis is an important mechanism for the occurrence and development of cancer. Consequently, flow cytometric assay was utilized to explore whether PIWIL2 could affect the apoptosis of glioma cells. The results demonstrated that the proportion of apoptotic cells in the PIWIL2-shRNA1-transfected U87 cells was significantly higher than that in the control group (Fig. 4A and B). Then, further investigation by western blotting assay was performed to compare the expression of PIWIL2 with some previously reported apoptosis-related molecules, such as cleaved caspase-3 and Bcl-2 (which is considered to be an antiapoptotic protein) $(23,24)$. The results revealed that knockdown of PIWIL2 increased the expression of active caspase-3, and inhibited the expression of Bcl-2 concomitantly (Fig. 4C and D). In conclusion, these results indicated 
A
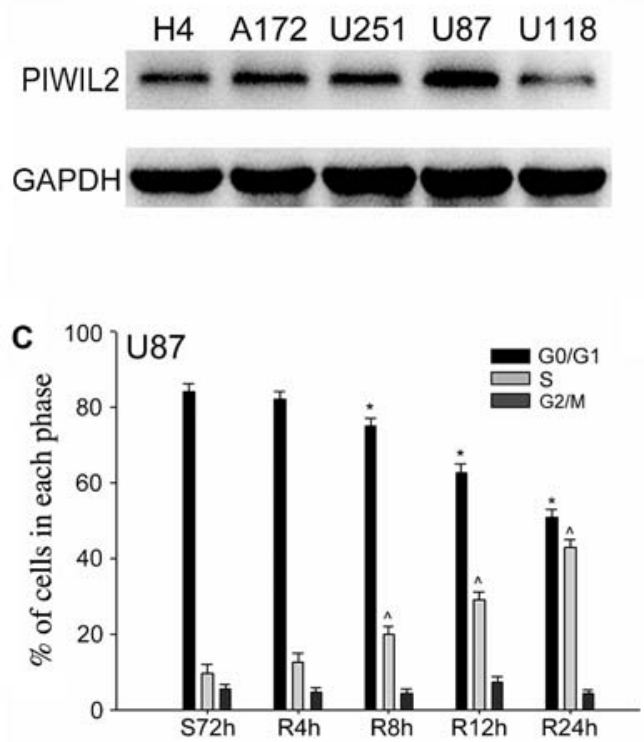

B

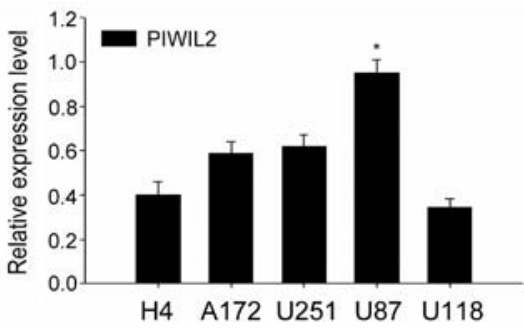

D

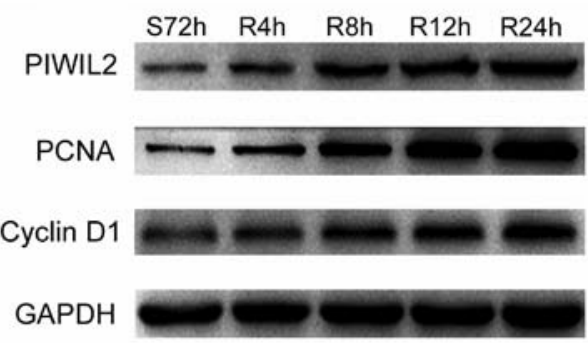

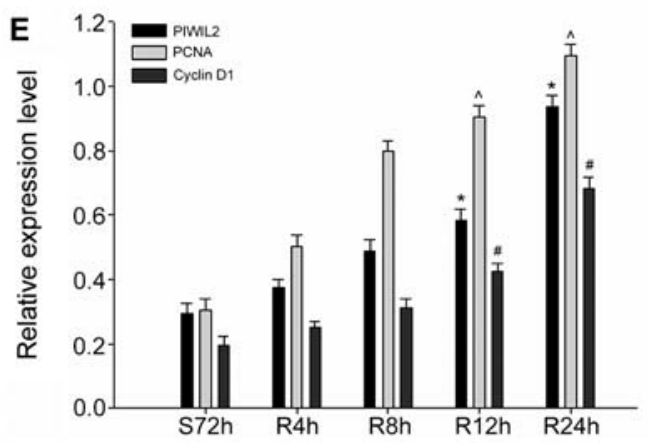

Figure 2. The expression of Piwi-like RNA-mediated gene silencing 2 (PIWIL2) and cell cycle-related molecules in glioma cells. (A and B) The expression level of PIWIL2 in five glioma cell lines (H4, A172, U251, U87 and U118); glyceraldehyde-3-phosphate dehydrogenase (GAPDH) was used as an internal standard. Data are expressed as the mean $\pm \operatorname{SEM}\left(n=3,{ }^{*} p<0.05\right)$. (C) Flow cytometry revealed the cell cycle progression of U87 cells in the serum starvation $(\mathrm{S})$ and re-feeding (R) model. Data are expressed as the mean \pm SEM $\left(n=3,{ }^{*}, p<0.05\right)$. (D and E) The expression of PIWIL2 and two cell cycle and proliferation markers (PCNA and cyclin D1) were detected concomitantly with western blot analysis at different time-points. GAPDH was used as a protein loading control. The ratio of PCNA and cyclin D1 compared to GAPDH was determined by densitometry, which is displayed in the form of a bar graph. The data are expressed as the mean \pm SEM $\left[n=3,{ }^{*, *} \mathrm{p} p<0.01\right.$, compared with the control cell serum-starved control for $\left.72 \mathrm{~h}(\mathrm{~S} 72 \mathrm{~h})\right]$.

that PIWIL2 may promote the development of glioma through its antiapoptotic effect.

PIWIL2 enhances glioma cell migration in vitro. Strong invasion and migration abilities are considered as significant characteristics of human gliomas. Moreover, previous studies have demonstrated that PIWIL2 promotes tumor cell migration (17,25). Based on this, we speculated that PIWIL2 may be associated to the migration of glioma cells. Through the wound-healing experiment, we observed that the migration of U87 cells transfected with PIWIL2-shRNA1 was significantly slower than that of the control group (Fig. 5A). In addition, the result of the Transwell experiment demonstrated that knockdown of the expression of PIWIL2 inhibited the ability of U87 cell migration to the bottom chambers (Fig. 5B). To further clarify the function of PIWIL2 in the migration of glioma cells, we compared the expression of certain migration-related molecules in the PIWIL2-shRNA1-transfected U87 and control cells. Notably, we determined that when PIWIL2 was silenced by shRNA1, the expression levels of two mesenchymal markers (N-cadherin and vimentin) were downregulated, while epithelial marker E-cadherin expression was upregulated (26). The results demonstrated that PIWIL2 may be involved in the epithelial-mesenchymal transition (EMT) of glioma cells. In addition, PIWIL2 was reported to promote the invasion and migration of colon and prostate cancer cells through the regulation of the expression of matrix metalloproteinase-9 (MMP9) $(17,25)$. In our study, we also observed that silencing of PIWIL2 decreased the expression of MMP9 (Fig. 5C and D). MMP9 is considered to be an important factor in the regulation of the migration of gliomas (27). Collectively the aforementioned experiments demonstrated that PIWIL2 was associated to the migration of gliomas.

\section{Discussion}

PIWIL2, as a member of the Piwi gene family, is considered to be closely related with RNA interference, stem cell self-renewal and translational regulation in germ cells (6). Moreover, it was also reported to be involved in the pathological process of the development and differentiation of cancer stem cells (CSCs) $(13,28)$. CSCs are regarded as the 
A

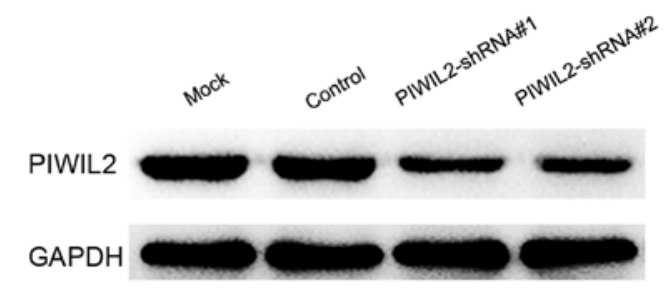

C
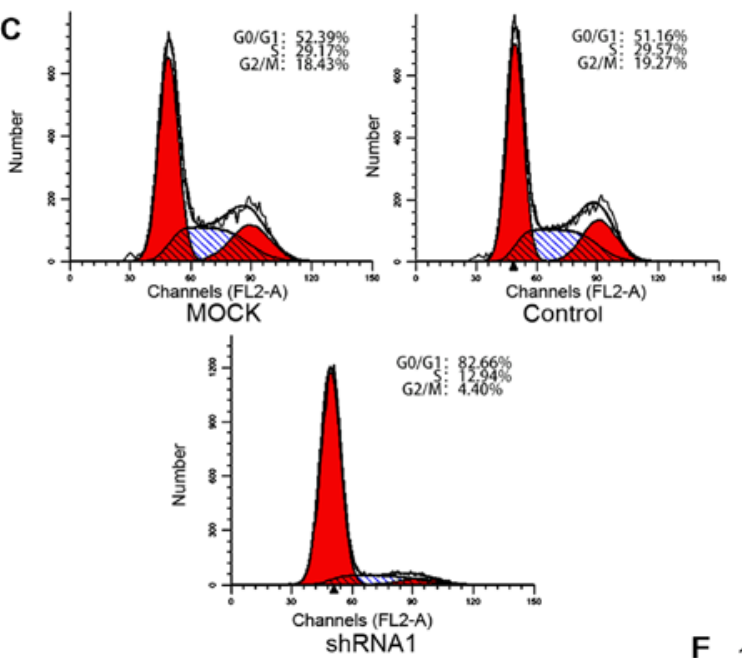

E

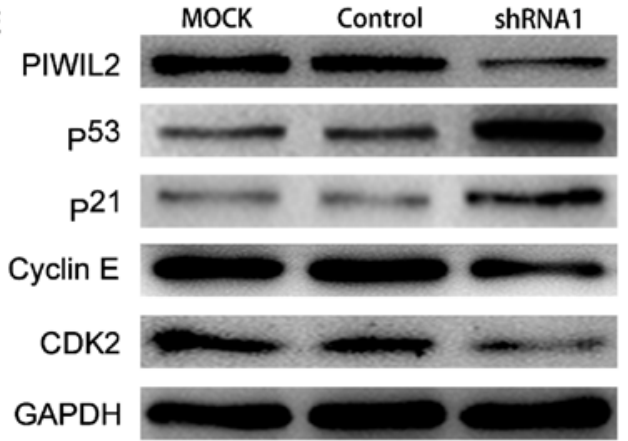

B
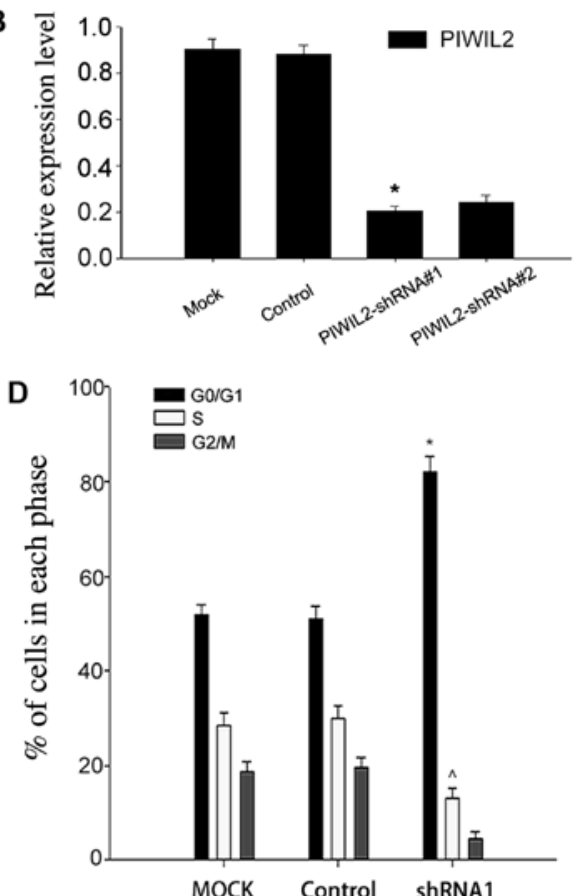

G

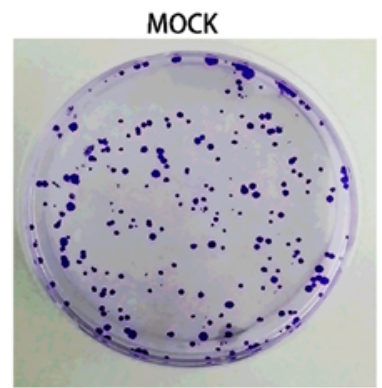

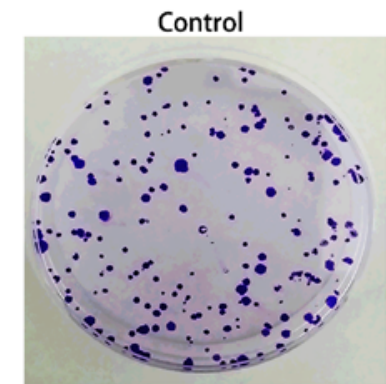
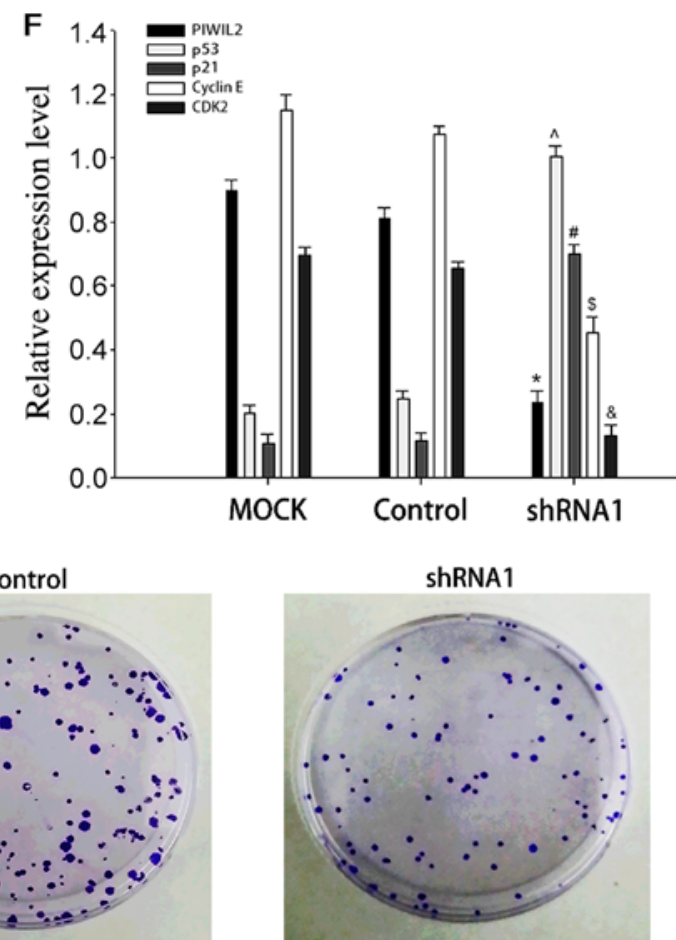

Figure 3. Knockdown of Piwi-like RNA-mediated gene silencing 2 (PIWIL2) inhibits the proliferation of U87 cells. (A and B) Detection of the expression of PIWIL2 in PIWIL2-shRNA-transfected-U87 cells by western blot analysis. The results revealed that PIWIL2-shRNA1 exhibited a stronger downregulation effect. Data are expressed as the mean \pm SEM ( $n=3,{ }^{*} p<0.05$ compared with the control groups). (C and D) PIWIL2-shRNA1-transfected U87 cells, control shRNA transfected U87 cells and untreated U87 cells were pretreated by serum starvation to maintain cell cycle synchronization. The cell cycle distribution was assessed by flow cytometry $48 \mathrm{~h}$ later. Data are expressed as the mean \pm SEM ( $\mathrm{n}=3$, ${ }^{*, n} \mathrm{p}<0.05$ compared with the control groups). (E and F) Western blot analysis revealed the influence of PIWIL2 knockdown on p53, p21, cyclin E and cyclin-dependent kinase 2 (CDK2) protein expression. Data are expressed as the mean $\pm \operatorname{SEM}\left(n=3,{ }^{*, *, t, \$, \&} \mathrm{p}<0.05\right.$ compared with the control groups). (G) Silencing of PIWIL2 inhibited cell growth as determined by colony formation assay.

major determinant of tumor initiation, propagation, metastasis and recurrence (29). Previous studies have demonstrated that PIWIL2 may be associated with the proliferation, invasion and migration of a variety of malignancies and predicts the poor prognosis of patients $(17,25)$. In this study, we investigated the potential role of PIWIL2 in the promotion of proliferation and migration of human gliomas, as well as demonstrated the clinical significance of its expression.

Through western blot and immunohistochemical analyses, we found that PIWIL2 was significantly overexpressed in 

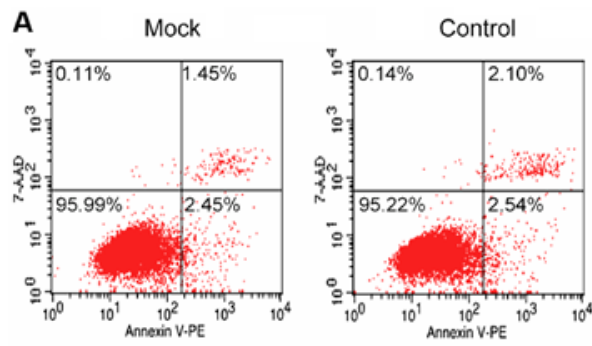

C

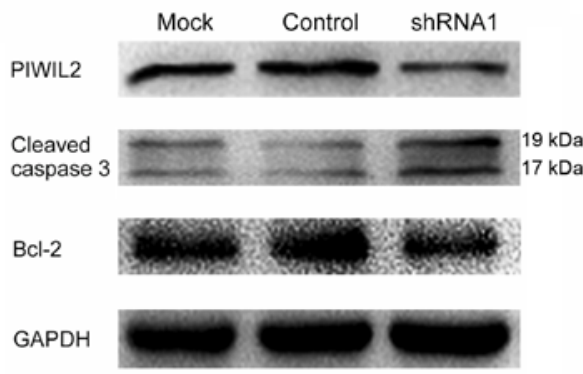

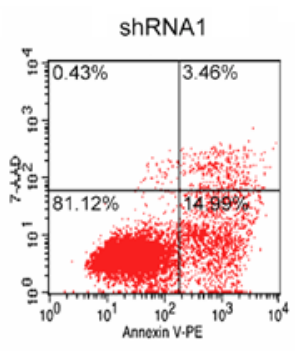
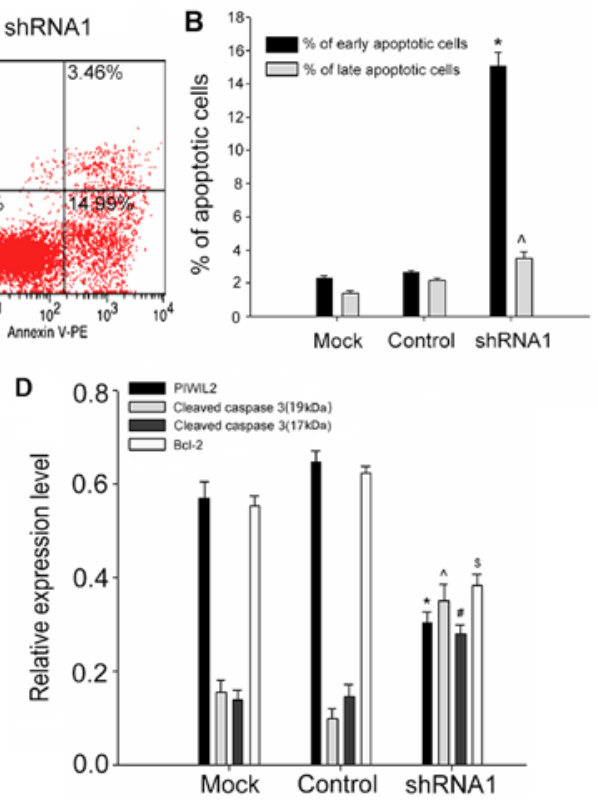

Figure 4. Knockdown effect of Piwi-like RNA-mediated gene silencing 2 (PIWIL2) on U87 cell apoptosis. (A and B) PE Annexin V apoptosis detection kit I and flow cytometer BD FACScan were used to detect the apoptosis of untreated U87 cells, PIWIL2-shRNA1-transfected U87 cells and control shRNAtransfected U87 cells. Data are expressed as the mean \pm SEM ( $n=3,{ }^{*, \wedge} p<0.05$ compared with the control groups). (C and D) The expression levels of apoptotic marker protein (cleaved caspase 3) and anti-apoptosis protein (Bcl-2) in all three experimental groups were detected by western blot analysis. Data are expressed as the mean \pm SEM ( $n=3,{ }^{*, N, \#, s} \mathrm{p}<0.05$ compared with the control groups).
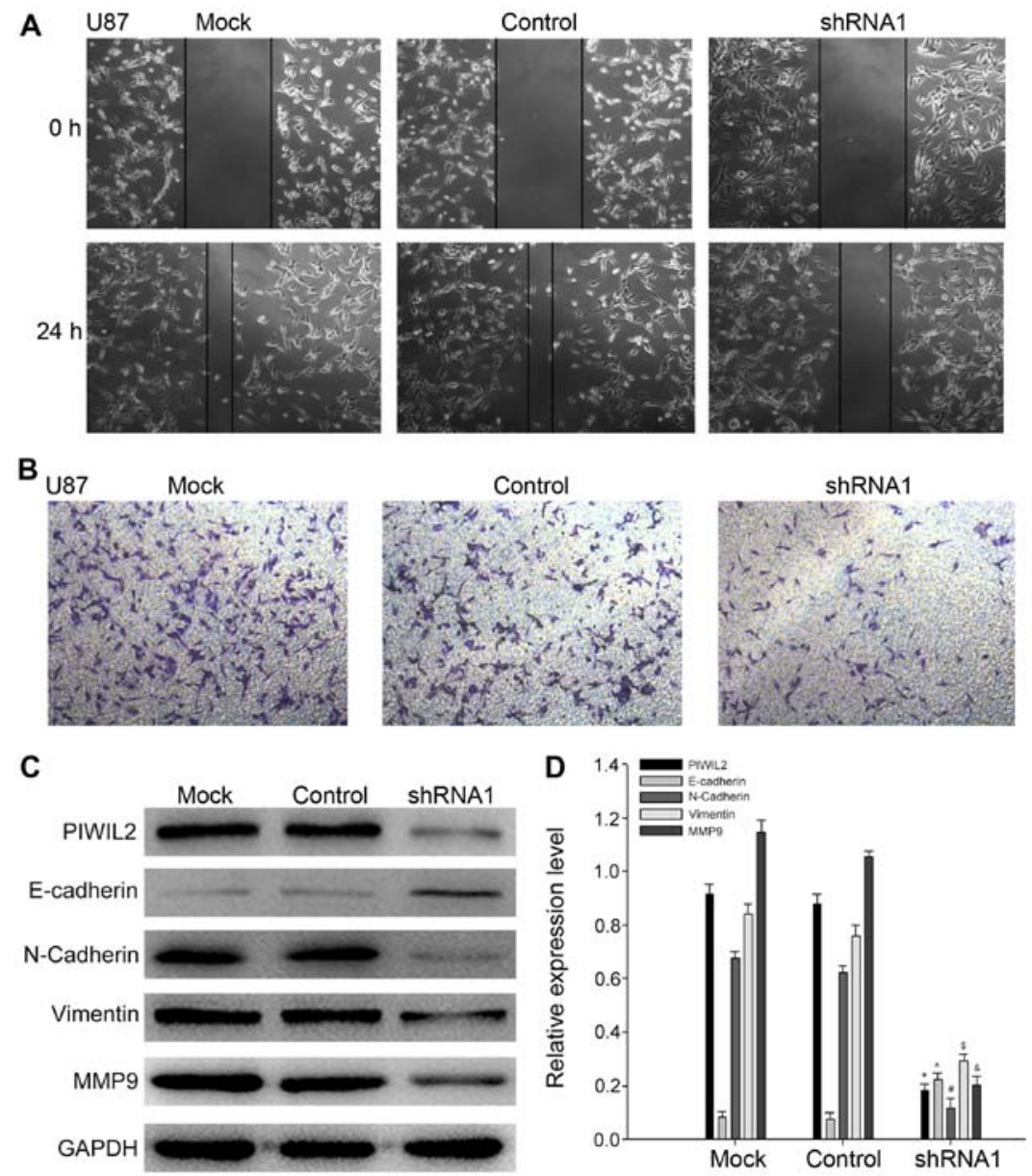

Figure 5. Silencing of Piwi-like RNA-mediated gene silencing 2 (PIWIL2) inhibits the migration of glioma cells. (A) Monolayer U87 cells transfected with control or PIWIL2 shRNA, as well as the mock group were analyzed by wound-healing assays. The migration of cells into the wound was assessed at 0 and $24 \mathrm{~h}$. (B) U87 cells were stained with crystal violet, and the migration ability of glioma cells was analyzed by Transwell experiment after $24 \mathrm{~h}$. (C and D) Western blot analysis of PIWIL2, E-cadherin, N-cadherin, vimentin and MMP9 levels in the mock, control and shRNA1 groups. Data are expressed as the mean $\pm \operatorname{SEM}\left(\mathrm{n}=3,{ }^{*, *, \$, \$, \&} \mathrm{p}<0.05\right.$ compared with the control groups). 
glioma tissues. Furthermore, the expression level of PIWIL2 exhibited an increasing trend, and was tightly associated with the increase in the pathological grade of the gliomas (WHO grade II-IV), which indicates that PIWIL2 may be involved in the pathological process of gliomas. Subsequently, the clinicopathological data of the corresponding patients with glioma were analyzed systematically. Through Kaplan-Meier analysis as well as univariate analysis by Cox proportional hazards model, it was revealed that the high expression of PIWIL2 indicated a poor prognosis with a significantly shorter overall survival.

From a macro point of view, the expression of PIWIL2 may contribute to the progression of gliomas. We further explored the effect of PIWIL2 on the proliferation and migration of human glioma cells in vitro. Cell cycle acceleration and apoptosis inhibition are considered to be important factors for the rapid proliferation of tumor cells $(30,31)$. Previous studies demonstrated that PIWIL2 could accelerate G1/S phase transformation of tumor cells (HeLa and HepG2 cells) by inducing c-Myc expression (22). In the current study, serum starvation and re-feeding experiments determined that the expression of PIWIL2 was gradually increased after serum readdition, consistent with two cell cycle-related proteins, PCNA and cyclin D1. Thus, we believe that PIWIL2 is involved in the progression of the cell cycle of glioma cells. In addition, flow cytometric analysis displayed that a higher proportion of U87 cells was arrested in the G0/G1 phase when the expression of PIWIL2 was silenced by RNAi technique. PIWIL2 was reported to function in the inhibition of p53 expression (11). p53, known as an important cancer-inhibiting protein, has an intimate connection with tumor cell cycle regulation and apoptosis (32). The p53 protein upregulates the expression of $\mathrm{p} 21$, which then blocks the cell cycle in the $\mathrm{S}$ phase by inhibiting the activation of the cyclin E/Cdk2 complex (33). Based on this theory, we found that silencing of PIWIL2 led to the upregulation of p53 and p21 expression, and the downregulation of the expression of cyclin $\mathrm{E}$ and $\mathrm{Cdk} 2$. These results demonstrated that PIWIL2 may induce glioma G1/S phase transition via dysregulation of the p53-p21-cyclin E/Cdk2 pathway. Moreover, flow cytometric analysis revealed that there was a significantly higher proportion of apoptotic cells in the PIWIL2-depleted U87 cells. PIWIL2 has already been demonstrated to inhibit cell apoptosis through the activation of the Stat3/Bcl-XL pathway (34). Similar to Bcl-XL, Bcl-2 and caspase 3 are also regulated by STAT3, which leads to cell apoptosis (35-37). In our study, we determined that downregulated expression of PIWIL2 led to the increased expression of cleaved caspase 3 while it inhibited Bcl-2 expression, which meant that PIWIL2 may inhibit glioma cell apoptosis by suppressing nuclear to mitochondrial translocation as well as the subsequent caspase activation.

Migration and invasion is another prominent feature of gliomas (38). In the study, wound healing and Transwell assays demonstrated that PIWIL2 promoted the migration of glioma cells. Previous studies have reavealed that EMT is an important process in which tumor cells can enhance their migration ability (39). Therefore, research concerning the effect of PIWIL2 on the EMT would be helpful to elucidate its mechanism in the migration of gliomas. Loss of cell-cell adhesion, increased tumor cell mobility, downregulated expression of epithelial markers and overexpression of mesenchymal markers are usually recognized as the main biological characteristics of EMT $(40,41)$. Our study revealed that inhibition of PIWIL2 expression in glioma cells increased epithelial marker E-cadherin expression, while it decreased the expression of mesenchymal markers N-cadherin and vimentin. Therefore, PIWIL2-induced EMT may be an important mechanism for the migration of glioma cells. Moreover, it was revealed that PIWIL2 promoted MMP9 expression by regulating its transcriptional activity, thereby enhancing the invasion and migration of colon cancer cells (25). MMP9 is also considered to be an important regulatory factor for the migration and invasion of gliomas (27). Western blot analysis demonstrated that silencing of PIWIL2 decreased the expression of MMP9 in U87 cells. Thus, we believe that the promotion of MMP9 expression was also one of the mechanisms through which PIWIL2 enhanced the migration of glioma cells. Nevertheless, specific mechanisms of PIWIL2 regulation on MMP9 expression, as well as the influence on its activity have yet to be elucidated through further experimental research. In conclusion, our research demonstrated that PIWIL2 is highly expressed in glioma tissues and provides important predictive information for patients with gliomas. Additionally, PIWIL2 was also positively correlated with the proliferation and migration of glioma cells. Nonetheless, the precise molecular mechanisms of PIWIL2 in gliomas require further investigation, with the hope to provide a new molecular target for the diagnosis and therapies of glioma in the future.

\section{Acknowledgments}

This study was supported by the National Natural Science Foundation of China (no. 81372687).

\section{References}

1. Wang Y, Liu F, Mao F, Hang Q, Huang X, He S, Wang Y, Cheng C, Wang $\mathrm{H}, \mathrm{Xu} \mathrm{G}$, et al: Interaction with cyclin $\mathrm{H} /$ cyclin-dependent kinase 7 (CCNH/CDK7) stabilizes C-terminal binding protein 2 (CtBP2) and promotes cancer cell migration. J Biol Chem 288: 9028-9034, 2013

2. Xu K, Pei H, Zhang Z, Dong S, Fu RJ, Wang WM and Wang $\mathrm{H}$ : FoxO3a mediates glioma cell invasion by regulating MMP9 expression. Oncol Rep 36: 3044-3050, 2016.

3. Batchelor TT, Reardon DA, de Groot JF, Wick W and Weller M: Antiangiogenic therapy for glioblastoma: Current status and future prospects. Clin Cancer Res 20: 5612-5619, 2014.

4. Tao T, Cheng C, Ji Y, Xu G, Zhang J, Zhang L and Shen A: Numbl inhibits glioma cell migration and invasion by suppressing TRAF5-mediated NF- $\mathrm{B}$ activation. Mol Biol Cell 23: 2635-2644, 2012

5. Hu B, Nandhu MS, Sim H, Agudelo-Garcia PA, Saldivar JC, Dolan CE, Mora ME, Nuovo GJ, Cole SE and Viapiano MS: Fibulin-3 promotes glioma growth and resistance through a novel paracrine regulation of Notch signaling. Cancer Res 72: 3873-3885, 2012.

6. Sasaki T, Shiohama A, Minoshima $S$ and Shimizu N: Identification of eight members of the Argonaute family in the human genome. Genomics 82: 323-330, 2003.

7. Höck J and Meister G: The Argonaute protein family. Genome Biol 9: 210, 2008.

8. Kuramochi-Miyagawa S, Kimura T, Ijiri TW, Isobe T, Asada N, Fujita Y, Ikawa M, Iwai N, Okabe M, Deng W, et al: Mili, a mammalian member of piwi family gene, is essential for spermatogenesis. Development 131: 839-849, 2004.

9. Cox DN, Chao A, Baker J, Chang L, Qiao D and Lin H: A novel class of evolutionarily conserved genes defined by piwi are essential for stem cell self-renewal. Genes Dev 12: 3715-3727, 1998. 
10. Chen $\mathrm{C}$, Liu $\mathrm{J}$ and $\mathrm{Xu}$ G: Overexpression of PIWI proteins in human stage III epithelial ovarian cancer with lymph node metastasis. Cancer Biomark 13: 315-321, 2013.

11. Lu Y, Zhang K, Li C, Yao Y, Tao D, Liu Y, Zhang S and Ma Y: Piwil2 suppresses p53 by inducing phosphorylation of signal transducer and activator of transcription 3 in tumor cells. PLoS One 7: e30999, 2012.

12. Chen L, Shen R, Ye Y, Pu XA, Liu X, Duan W, Wen J, Zimmerer J, Wang Y, Liu Y, et al: Precancerous stem cells have the potential for both benign and malignant differentiation. PLoS One 2: e293, 2007.

13. Lee JH, Schütte D, Wulf G, Füzesi L, Radzun HJ, Schweyer S, Engel W and Nayernia K: Stem-cell protein Piwil2 is widely expressed in tumors and inhibits apoptosis through activation of Stat3/Bcl-XL pathway. Hum Mol Genet 15: 201-211, 2006.

14. Liu JJ, Shen R, Chen L, Ye Y, He G, Hua K, Jarjoura D, Nakano T, Ramesh GK, Shapiro CL, et al: Piwil2 is expressed in various stages of breast cancers and has the potential to be used as a novel biomarker. Int J Clin Exp Pathol 3: 328-337, 2010.

15. Qu X, Liu J, Zhong X, Li X and Zhang Q: PIWIL2 promotes progression of non-small cell lung cancer by inducing CDK2 and cyclin A expression. J Transl Med 13: 301, 2015.

16. Wang Y, Liu Y, Shen X, Zhang X, Chen X, Yang C and Gao H: The PIWI protein acts as a predictive marker for human gastric cancer. Int J Clin Exp Pathol 5: 315-325, 2012.

17. Yang Y, Zhang X, Song D and Wei J: Piwil2 modulates the invasion and metastasis of prostate cancer by regulating the expression of matrix metalloproteinase-9 and epithelial-mesenchymal transitions. Oncol Lett 10: 1735-1740, 2015.

18. Oh SJ, Kim SM, Kim YO and Chang HK: Clinicopathologic implications of PIWIL2 expression in colorectal cancer. Korean J Pathol 46: 318-323, 2012.

19. Mu H, Lin KX, Zhao H, Xing S, Li C, Liu F, Lu HZ, Zhang Z, Sun YL, Yan XY, et al: Identification of biomarkers for hepatocellular carcinoma by semiquantitative immunocytochemistry. World J Gastroenterol 20: 5826-5838, 2014

20. Preusser M,Hoeftberger R, Woehrer A, Gelpi E, Kouwenhoven M, Kros JM, Sanson M, Idbaih A, Brandes AA, Heinzl H, et al: Prognostic value of Ki67 index in anaplastic oligodendroglial tumours - a translational study of the European Organization for Research and Treatment of Cancer Brain Tumor Group. Histopathology 60: 885-894, 2012.

21. Darzynkiewicz Z, Zhao H, Zhang S, Lee MY, Lee EY and Zhang Z: Initiation and termination of DNA replication during $\mathrm{S}$ phase in relation to cyclins $\mathrm{D} 1, \mathrm{E}$ and $\mathrm{A}, \mathrm{p} 21^{\mathrm{WAF} 1}, \mathrm{Cdt} 1$ and the p12 subunit of DNA polymerase $\delta$ revealed in individual cells by cytometry. Oncotarget 6: 11735-11750, 2015.

22. Yao Y, Li C, Zhou X, Zhang Y, Lu Y, Chen J, Zheng X, Tao D, Liu Y and Ma Y: PIWIL2 induces c-Myc expression by interacting with NME2 and regulates c-Myc-mediated tumor cell proliferation. Oncotarget 5: 8466-8477, 2014.

23. Yang S, Wang L and Kong Q: Depression of focal adhesion kinase induces apoptosis in rat osteosarcoma OSR- 6 cells in a caspase-dependent pathway. Cell Biochem Biophys 70: 765-770, 2014.

24. Al-Shenawy HA: Expression of beclin-1, an autophagy-related marker, in chronic hepatitis and hepatocellular carcinoma and its relation with apoptotic markers. APMIS 124: 229-237, 2016.

25. Li D, Sun X, Yan D, Huang J, Luo Q, Tang H and Peng Z: Piwil2 modulates the proliferation and metastasis of colon cancer via regulation of matrix metallopeptidase 9 transcriptional activity. Exp Biol Med (Maywood) 237: 1231-1240, 2012.

26. Tian L, Lu ZP, Cai BB, Zhao LT, Qian D, Xu QC, Wu PF, Zhu Y, Zhang JJ, Du Q, et al: Activation of pancreatic stellate cells involves an EMT-like process. Int J Oncol 48: 783-792, 2016.
27. Veeravalli KK and Rao JS: MMP-9 and uPAR regulated glioma cell migration. Cell Adhes Migr 6: 509-512, 2012.

28. Litwin M, Dubis J, Arczyńska K, Piotrowska A, Frydlewicz A, Karczewski M, Dzięgiel P and Witkiewicz W: Correlation of HIWI and HILI expression with cancer stem cell markers in colorectal cancer. Anticancer Res 35: 3317-3324, 2015.

29. Akbari-Birgani S, Paranjothy T, Zuse A, Janikowski T, Cieślar-Pobuda A, Likus W, Urasińska E, Schweizer F, Ghavami $\mathrm{S}$, Klonisch T, et al: Cancer stem cells, cancer-initiating cells and methods for their detection. Drug Discov Today 21: 836-842, 2016.

30. Bunt J, de Haas TG, Hasselt NE, Zwijnenburg DA, Koster J, Versteeg R and Kool M: Regulation of cell cycle genes and induction of senescence by overexpression of OTX 2 in medulloblastoma cell lines. Mol Cancer Res 8: 1344-1357, 2010.

31. Jiang C, Song T, Li J, Ao F, Gong X, Lu Y, Zhang C, Chen L, Liu Y, He H and Huang O: RAS promotes proliferation and resistances to apoptosis in meningioma. Mol Neurobiol: 779-787, 2017.

32. Liu H, Chen F, Zhang L, Zhou Q, Gui S and Wang Y: A novel all-trans retinoic acid derivative 4-amino 2 trifluoromethyl-phenyl retinate inhibits the proliferation of human hepatocellular carcinoma HepG2 cells by inducing G0/G1 cell cycle arrest and apoptosis via upregulation of p53 and ASPP1 and downregulation of iASPP. Oncol Rep 36: 333-341, 2016.

33. Zolota V, Sirinian C, Melachrinou M, Symeonidis A and Bonikos DS: Expression of the regulatory cell cycle proteins $\mathrm{p} 21$, p27, p14, p16, p53, mdm2, and cyclin $\mathrm{E}$ in bone marrow biopsies with acute myeloid leukemia. Correlation with patients' survival. Pathol Res Pract 203: 199-207, 2007.

34. Lee JH, Jung C, Javadian-Elyaderani P, Schweyer S, Schütte D, Shoukier M, Karimi-Busheri F, Weinfeld M, Rasouli-Nia A, Hengstler JG, et al: Pathways of proliferation and antiapoptosis driven in breast cancer stem cells by stem cell protein piwil2 Cancer Res 70: 4569-4579, 2010.

35. Dlamini Z, Rupnarain C, Naicker S, Hull R and Mbita Z: Expression analysis and association of RBBP6 with apoptosis in colon cancers. J Mol Histol 47: 169-182, 2016.

36. Jin H, Chen H, Yu K, Zhang J, Li B, Cai N and Pan J: Resveratrol inhibits phosphorylation within the signal transduction and activator of transcription 3 signaling pathway by activating sirtuin 1 in SW1353 chondrosarcoma cells. Mol Med Rep 14: 2685-2690, 2016

37. Baral R, Bose A, Ray C, Paul S, Pal S, Haque E, Mishra B, Pal D, Nagpal JK, Panda CK, et al: Association of early phase of colorectal carcinogenesis with STAT3 activation and its relevance in apoptosis regulation. Exp Mol Pathol 87: 36-41, 2009.

38. Liang Q, Ma C, Zhao Y, Gao G and Ma J: Inhibition of STAT3 reduces astrocytoma cell invasion and constitutive activation of STAT3 predicts poor prognosis in human astrocytoma. PLoS One 8: e84723, 2013

39. Song X, Han P, Liu J, Wang Y, Li D, He J, Gong J, Li M, Tu W, Yan $\mathrm{W}$, et al: Up-regulation of SPOCK1 induces epithelial-mesenchymal transition and promotes migration and invasion in esophageal squamous cell carcinoma. J Mol Histol 46: 347-356, 2015.

40. Chen HN, Yuan K, Xie N, Wang K, Huang Z, Chen Y, Dou Q, Wu M, Nice EC, Zhou ZG, et al: PDLIM1 stabilizes the E-cadherin/ $\beta$-catenin complex to prevent epithelial-mesenchymal transition and metastatic potential of colorectal cancer cells. Cancer Res 76: 1122-1134, 2016.

41. Tiwari N, Gheldof A, Tatari M and Christofori G: EMT as the ultimate survival mechanism of cancer cells. Semin Cancer Biol 22: 194-207, 2012. 